\title{
Cognitive Behavioural Therapy Techniques for Treating Verbal Impair- ments in Children Diagnosed with Autism Spectrum Disorder - A Review
}

Afreen Ahmad ${ }^{1}$, Sanna Huda ${ }^{1}$, Imaan Kherani ${ }^{1}$, Zeba Khoja ${ }^{1}$, Jasmine Nanji ${ }^{1}$

1. McMaster University

Abstract

This paper aims to provide an overview of current research in cognitive behavioural interventions which address verbal impairment in children with autism. The studies are evaluated based on methodological quality and the validity of the data collected. Studies examining behavioural interventions for children with autism were selected from a number of databases, namely Ovid, PsychINFO, and Embase. Multiple filtration rounds were conducted to ensure that papers met the inclusion criteria, followed the DSM IV autism definition, and met the methodological quality standards. A CONSORT style observational longitudinal checklist was used to evaluate the methodological quality of the studies. Criteria pertaining to study designs were more commonly addressed than those focusing on internal validity. Analysis of literature subsequent to the year 2000 demonstrated an emergence of behavioural therapies focused on remediating verbal impairment in children diagnosed with autism. Common limitations amongst all reviewed papers were discussed in terms of impact on validity and reliability. Finally, the discussion consolidated the future directives noted in all papers to discuss trajectories for further research. The chosen literature often neglected to include essential quantitative information that affected their validity. Inclusion of control groups and appropriate sample sizes should be investigated, and future directions of this research should include the use of a diverse sample that is representative of different ethnic backgrounds and socioeconomic statuses

\section{Key Words}

Autism, cognitive behavioural therapy, methodological quality, verbal impairments, children

\section{Introduction}

The DSM IV defines autism as a neurodevelopmental disorder that significantly hinders ones' social abilities (1). Although limitations to verbal and social skills vary on an individual basis, communicative skills are often characterized by repetitive tendencies, restrictive socio-emotional interactions, and impaired speech (2).
Consequently, individuals with autism experience difficulty maintaining positive relationships due to delayed speech and an inability to interpret nonliteral language and emotion. Unfortunately, in many cases, these symptoms and behaviors worsen during adolescence $(2,3)$. Management of auxiliary symptomatology has been a focus of current autism research to improve quality of life, social interactions, and psychological well-being for autistic individuals. Specifically, current research is focused on improving verbal impairment of autistic individuals in hopes of improving sociability. Laugeson notes that ameliorated verbal competency increases the number of treatment options available to the patient for secondary symptoms (4).

Behavioural therapy, a branch of psychotherapy, is often pursued in research to manage autism's auxiliary symptoms as its holistic approach analyzes environmental influences on specific behavioural patterns. Behavioural therapy can be classified into four subcategories: positive behaviour support, applied behavioural analysis, cognitive behavioural therapy, and the teaching family model (5-7). Positive behaviour support employs conditioning and reinforcement to challenge existing behavioural patterns. Applied behavioural analysis addresses social and communication deficits (5-7). Cognitive behavioural therapy seeks to produce change in the subject's emotions and perceptions using social prompted interaction and technological intervention $(5,6)$. Finally, the teaching family model is used to rehabilitate children in a residential setting (8).

Studies involving behavior therapy employ a longitudinal approach to monitor the interventions' effectiveness, and the participants' behavioural improvements over time. However, the incidence, social and demographic variability, societal perceptions, and nature of these diagnoses renders valid and generalizable research in this field difficult to execute $(9,10)$. Literature focused on autism is often limited in terms of sample size, generally involving male study populations with access to social and economic health care interventions. As research continues to progress in this field, it is important to evaluate the methodological quality of current and existing research, and provide recommendations to improve 
future research (10).

To address the above conflict, this paper aims to evaluate the methodological quality of research on behavioural therapies designed to manage verbal impairment in children with autism. The reviewed studies were published between 2000 and 2018, and regard children up to, and including age seventeen. A literature search was conducted where methodologically sound, longitudinal studies scoring at least $17 / 33$ on the CONSORT style checklist were critiqued (9). Our objective was to analyze the discrepancies in current research regarding gender, racial and socioeconomic biases that affected external validity. Further, the lack of quantitative analysis was examined regarding the effect on validity of conclusions drawn from the included literature. It is hoped that the discrepancies discussed will provide recommendations for methodological improvement of future research in the management of autism related verbal impairment.

\section{Methods}

Peer-reviewed literature was extracted from Embase, PsychINFO, and OVID databases using a string of keywords: "all(Children ASD Autism Cognitive Behaviour Therapies; verbal)." American and Canadian spellings were taken into account for "behaviour" and "behavior" which yielded no significant change in the number of results found. In addition, reference lists of primary articles were manually investigated to conduct a more thorough search.

To meet our inclusion criteria, the paper had to have a focus on ASD and cognitive behavioural therapy approaches. Documents that were published before January 2000, after October 2017, had subjects aged 18 and above, or were not published in English were excluded. The 86 results found from the primary search were filtered using this inclusion/exclusion criteria, leaving 5 papers. The limited number of studies suggested that cognitive behavioural therapy is still in its infancy. The paper was redirected to follow the DSM IV autism definition under ASD. Additionally, the research question was redefined to include the other three types of behavioural therapy in order to enlarge our scope of research. Although our exclusion criteria remained the same, our new inclusion criteria was adapted to include all conditions under ASD, and included all forms of behavioural therapies. Once the databases were reexamined, 17 additional papers were found. The remaining studies' methodological quality was then assessed using an adapted form of a CONSORT checklist specific to longitudinal studies (9). A CONSORT style observational longitudinal checklist was specifically used to evaluate the methodological quality of the studies. This CONSORT style checklist is a remodeled checklist which al- lowed for the inclusion of all applicable studies, as well as the use of a reliable criteria checklist to assess validity. This criterion was evaluated to be an effective checklist as depicted by its use in a study by Tooth el al. (9).

The 17 papers that met the inclusion criteria were subjected to the CONSORT style checklist and were given a final score according to the number of criteria they satisfied (out of a total of 33). Papers scoring 17 and above were selected, since that was the average score of the papers included in the Tooth et al. study. A total of nine papers met the checklist cutoff score (Figure 1). Only papers that had above average scores (a score of 17 or higher) were selected in order to focus on the major limitations in validity.

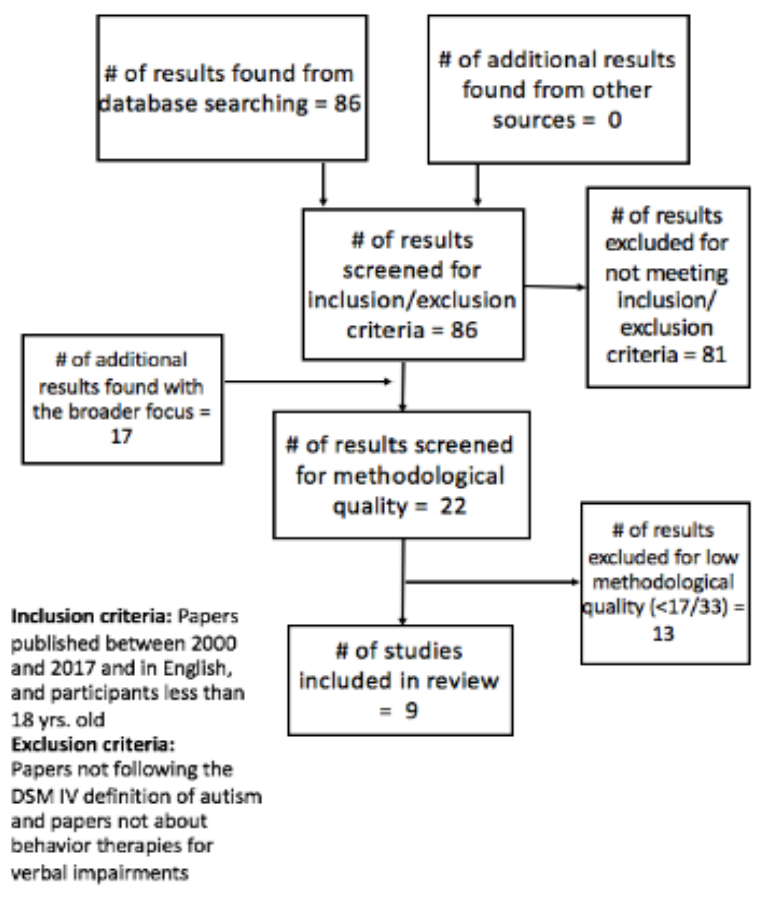

Figure 1: Chart of filtering process for inclusion in this review.

\section{Results}

The CONSORT style checklist criteria with the highest satisfaction rate (90-100\%) were as follows: clearly stated objectives/ hypotheses of the study, defined sampling frames, defined study populations, stated location/venue of the study, stated eligibility criteria, and described data collection methods. The most poorly met criteria (satisfaction rate of $20 \%$ and below) were reported numbers for how many participants did or did not consent to the study, stated reasons for refusal to consent, comparison between consenters and non-consenters, and analyses that took missing 
Table I: Percentage of papers that met each of the checklist criteria.

\begin{tabular}{|c|c|}
\hline Criteria & $\begin{array}{c}\% \text { of Papers that Met Each Section of the } \\
\text { Criteria }\end{array}$ \\
\hline Statement of objectives and hypothesis & 100 \\
\hline Definition of target population & 77 \\
\hline Definition of sampling frame & 100 \\
\hline Definition of study population & 100 \\
\hline Study setting & 100 \\
\hline Dates of study & 66 \\
\hline Eligibility criteria & 100 \\
\hline Mention of "Selection in" issue & 44 \\
\hline Justification of number of participants & 33 \\
\hline Discussion of eligibility criteria & 44 \\
\hline Discussion of ineligibility & 33 \\
\hline Numbers of consent reported & 11 \\
\hline Reasons for refusal of consent & 0 \\
\hline consenters compared with non-consentors & 11 \\
\hline Number of participants at the beginning of the study & 88 \\
\hline Data collection method & 100 \\
\hline Reliability of measurement methods & 88 \\
\hline Validity of measurement methods & 66 \\
\hline Discussion of confounders & 44 \\
\hline Number participants at each stage included & 77 \\
\hline Explanation of loss to follow up & 22 \\
\hline Missingness/ of data at each stage & 33 \\
\hline Type of analysis conducted & 88 \\
\hline Longitudinal analysis & 33 \\
\hline Absolute effect sizes & 33 \\
\hline Relative effect sizes & 44 \\
\hline Follow-up & 33 \\
\hline Confounders accounted for in analysis & 33 \\
\hline Missing data in analysis & 11 \\
\hline Biases assessed qualitatively & 66 \\
\hline Biases estimated quantitatively & 44 \\
\hline Refer to target population & 88 \\
\hline Discussion of generalizability & 88 \\
\hline
\end{tabular}


data into account.

Five individuals rated the papers to determine validity using the CONSORT style checklist criteria (10). Rating was standardized via an initial pre-rating discussion of what satisfied the criteria, and a final post-rating discussion comparing all final ratings and how they were determined.

In accordance with the results of Tooth et al.'s study, an analysis of our selected papers indicated that the criteria relating to study design were more highly reported than the criteria involving internal validity (10). Internal validity is ensuring that no other variable except the one studied is the cause of the result, whereas external validity is the generalizability of the results to the population. In order to address this pitfall in methodological quality, observational longitudinal studies should satisfy a list of internal and external validity criteria and should follow the flow diagram drafted by Tooth et al. (Figure 2).

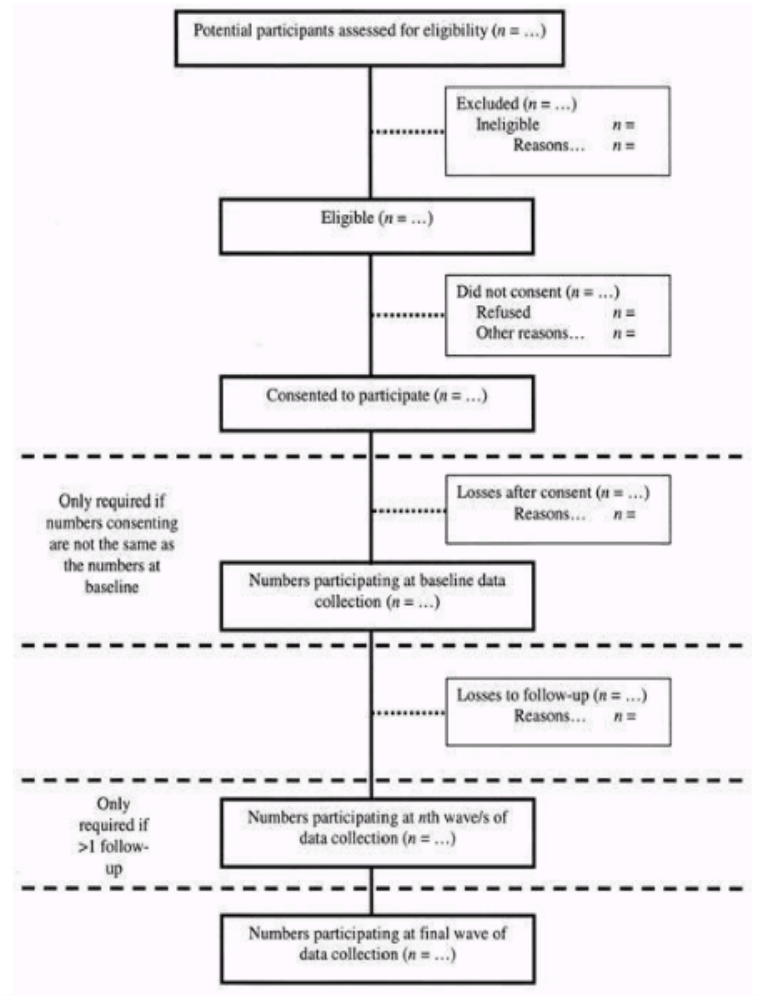

Figure 2: Flowchart outlining steps to improve internal and external validity in observational longitudinal studies (10).

MEHRIT is a social-interaction based therapeutic technique that was seen to improve verbal impairment in pediatric cases of autism (11). MEHRIT focuses on social interaction through therapy sessions that are based on the premise of play. The study followed 51 children subjected to two hours of therapy per week and three hours of interaction with the child per day. The chil- dren were categorized by language capabilities and split into two groups - one for MEHRIT and the other for community treatment (CT) (11). The dependent variables measured included commenting, labeling, responding, directing, sharing, obtaining information, rejecting or protesting, social conventions, and spontaneous social expression. The CONSORT style checklist was not met in the following categories: definition of target population, dates of study, justification of number of participants, discussion of eligibility criteria, discussion of ineligibility, number of consent reported, reasons for refusal of consent, consenters compared with non-consenting, discussion of confounders, missingness of data at each stage, longitudinal analysis, absolute effect size, confounders accounted for in analysis, and missing data in analysis. The MERHIT study did not stratify their study participants by race and gender (11).

The clinical research conducted by Green et al. was composed of 152 children diagnosed with autism between the ages of two years and four years, eleven months (12). Each parent was paired with a therapist to create an individualized treatment plan based on the Preschool Autism Communication Trial (PACT) model that focused on improving the communicative skills of the participants. The PACT model consisted of individual family sessions with a therapist. Of the participants, 75 were assigned to PACT and 77 remained on their regular treatment (TAU) (12). The three dependent variables in this study included parent-child interaction, child language and social communication, and adaptive functioning within the school. The CONSORT style checklist was not met in the following categories: justification of number of participants, discussion of eligibility criteria, discussion of ineligibility, number of consent reported, reasons for refusal of consent, consenters compared with non-consenters, validity of measurement methods, discussion of confounders, explanation of loss to follow up, relative effect sizes, follow up, missing data in analysis, biases asses qualitatively, and the discussion of generalizability. This study took place in London, Manchester and Newcastle, where 8\% of the participants participating in PACT were female (12). The ethnicities of these families were noted; London (35\% both white, $4 \%$ mixed, $62 \%$ non-white), Manchester (62\% white, $8 \%$ mixed, $19 \%$ non-white), and Newcastle ( $84 \%$ white, $8 \%$ mixed, $8 \%$ nonwhite). Furthermore, the socioeconomic status of the families was mentioned, where at least one parent was in a professional or administrative occupation (London (65\%), Manchester (69\%) and Newcastle (64\%)) (12).

The Picture Exchange Communication System (PECS), a cognitive intervention for nonverbal children with autism, was imple- 
mented in a study by Howlin et al. (13). Its goal was to develop spontaneous social-communication skills through the use of tools (symbols), and techniques (reinforcements) (13). Children were taught to request objects using specific symbols. Correct use of these symbols resulted in the item being given to the participants as a form of positive reinforcement, allowing participants to select and exchange symbols for objects (13). Post treatment, children's speech initiations and symbol use increased (13). Additionally, the ADOS-G Communication and Reciprocal Social Interaction domain scores demonstrated a delayed progression of autistic symptoms in the treatment group that received immediate therapy (13). The study had 84 participants who were split into three groups: immediate treatment group ( $80 \%$ male, $20 \%$ female), delayed treatment group ( $90 \%$ male, $10 \%$ female), and no treatment group (89\% male, 11\% female) (13). The paper does not mention the ethnic backgrounds of its participants. Since participants were recruited from fifteen different schools in the UK, based on lack of exposure to PECS, there was no sizeable socioeconomic divide (13). The CONSORT style checklist criteria that was not met include dates of study, numbers of consent reported, reasons for refusal of consent, consenters compared to non-consenters, number of participants at the beginning of the study, reliability of measurement methods, validity of measurement methods, number of participants at each stage, explanation of loss to follow up, missing data at each stage, and longitudinal analysis (13).

The script-fading procedure was examined and implemented by Garcia-Albea et al. to examine the initiation of spontaneous speech without the use of external prompts for children with autism (14). The study involved a multiple-probe design where participants were trained with several toys, then presented with novel toy prompts with hopes to increase the frequency of novel interactions. Types of social interactions, body language, and unscripted initiations were also measured with the study's dependent variable, evaluating the extent of language replication between the participant and the prompter (14). The sample size involved four boys clinically diagnosed with autism ranging from the ages of four to six, all scoring a lower equivalent age on the Preschool Language Scale. No break down on race, or socioeconomic status was provided, although all participants had access to one-on-one ongoing supervision and school-based interventions (14). Although pre-and post-probe session data was collected, no control group was included. Based on the CONSORT style checklist adapted for longitudinal research, the study scored a 19/32, meeting the average as defined by Tooth et al. The paper did not include information on eligibility, ineligibility, a justification for the number of partici- pants, "selection in" issues, or reasons for consent refusal. In the analysis stage, the reasons for loss to follow up were not discussed, or quantified, nor was effect size taken account. Finally, the impact of biases and confounders were not estimated quantitatively, although they were discussed.

The VocSyl study by DeThorne L produced visual feedback through computerized technologies to facilitate the speech-language development in children (15). The effectiveness of two speech-language interventions on children's multisyllabic productions was tested through visual inspection comparing the study group to the control group (14). VocSyl, the first intervention, introduced a computerized system that electronically produced a visualization of the attempted syllables in the form of shapes. A second intervention used a traditional, non-computerized Pacing Board to slow the participants' speech by encouraging them to break down the syllables of each word (15). Of the eighteen participants, six were clinically diagnosed with autism. Twelve were male, and fifteen were identified by their parents as White/ Caucasian (1 as African American/Caucasian, 1 as Hispanic/Caucasian, and 1 as Hispanic/Latino) (15). The CONSORT style checklist was not met in the following categories: mention of "selection in" issue, justification of number of participants, discussion of eligibility criteria, discussion of ineligibility, numbers of consent reported, reasons for refusal of consent, consenters compared with non-consenters, discussion of confounders, missingness of data at each stage, longitudinal analysis, absolute effect sizes, relative effect sizes, follow-up, confounders accounted for in analysis, missing data in analysis, and biases estimated quantitatively (15).

The study by Rogers et al. involved ten children showing signs of verbal impairment and included two interventions: the Denver Model; a behavioural and relationship-oriented speech intervention, and PROMPT; a neuro-developmental speech impairment therapy (16). The number of functional words used per session was charted and visually inspected as the analyzed measure. The twelve treatment sessions lasted one hour per week, in addition to the daily one hour sessions facilitated by parental figures. Following twelve treatment hours, 80 percent of children showed an increase in the frequency of speech, or an expanded vocabulary size (16). The study concluded that participants with a lower severity of symptoms had a better response to both models since motor imitation and attention skills were required for functional use (16). All ten participants were male, $80 \%$ were Caucasian, $10 \%$ were Hispanic/Biracial, and $10 \%$ were African-American. Due to the study's single subject design, each participant served as their own control. The CONSORT style checklist criteria that was not met 
by this study include mention of "selection in" issue, discussion of ineligibility, numbers of consent reported, reasons for refusal of consent, validity of measurement methods, number of participants included at each stage, explanation to loss to follow up, type of analysis conducted, longitudinal analysis, absolute effect sizes, relative effect sizes, confounders accounted for in analysis, missing data in analysis, and biases assessed qualitatively (16).

Charlop-Christy and Kelso aimed to assess the efficacy of script and cue card prompted conversational speech in children with autism. The study population consisted of three boys between the ages of eight and eleven who attended a behavioral treatment program twice a week (17). Conversations were based on topics presented by the scripted cue card and focused on abstract concepts relating to school activities and favourite pastimes tailored to participants' interests. Significant improvement of conversational ability was noticed in majority of the participants, as conversation frequency increased and unfamiliar discussion topics were explored (17). This intervention resulted in all participants increasing in informal speech ability. It was concluded that after two to four practices using cues cards, the children were able to collectively respond to two consecutive conversations (17). The subject's verbal abilities were measured at baseline and again after treatment, allowing them to serve as their own controls (17). There was no comment about the participants' socioeconomic or ethnic backgrounds (17). The CONSORT style checklist criteria that was not met by this study include justification of number of participants, discussion of eligibility criteria, discussion of ineligibility, numbers of consent reported, reasons for refusal of consent, consenters compared with non-consenters, explanation of loss to follow up, absolute effect sizes, relative effect sizes, follow-up, confounders accounted for in analysis, missing data in analysis, and biases estimated quantitatively.

Carter et al. conducted a randomized control trial using 62 participants and their parents to analyze the effects of Hanen's 'More than Words' (HMTW) program compared to a control group that continued to receive its traditional therapies (18). HMTW is a training program facilitated by parents to support, educate, and enhance the skills of their autistic children (18). The program was comprised of eight weekly group sessions and three individual sessions attended by the families of the participants. The overarching purpose of the training program was to encourage parent driven, playful interaction with their children to promote the development of communication skills (18). The subjects in this study were ethnically diverse, with a predominantly male population. Parents identified $47.4 \%$ of children as White, $38.6 \%$ as
Hispanic or Latino, 3.5\% as Black, 5.3\% as Asian/White, 3.5\% as American Indian/Alaskan Native/White and $1.8 \%$ as American Indian/Alaskan (18). The CONSORT style checklist was not met in the following categories: dates of study, mention of "selection in" issue, reasons for refusal of consent, consenters compared with non-consenters, discussion of confounders, explanation of loss to follow up, missingness of data at each stage, longitudinal analysis, absolute effect sizes, loss to follow-up, confounders accounted for in analysis, missing data in analysis, biases assessed qualitatively, biases estimated quantitatively, and reference to target population.

Studer et al. conducted a study on the effectiveness of early intensive behavioural intervention (EIBI) for children with autism, in addition to providing an overview of its current use in both the United States of America and Switzerland. EIBI is a 40 hour per week, comprehensive behavioural therapy that focuses on treating patients under the age of four (19). The study took place between April 2004 and March 2014 and followed 23 participants aged five and below. $17.4 \%$ of the participants had two Swiss parents, 39.1\% had one Swiss parent, and $43.5 \%$ had no Swiss parents (19). The CONSORT style checklist was not met in the following categories: definition of target population, mention of "selection in" issue, justification of number of participants, numbers of consent reported, reasons for refusal of consent, consenters compared with non-consenters, discussion of confounders, explanation of loss to follow up, missingness of data at each stage, longitudinal analysis, follow-up, confounders accounted for in analysis, missing data in analysis, and biases estimated quantitatively. The authors commented on the financial and social constraints of behavioural therapy, highlighting attention to cultural barriers, such as language. Moreover, the longevity of this research, as the intervention model must be individualized and adapted to each case of autism, creates substantial financial barriers that must be considered prior to beginning research (19). Some of the limitations included not having a methodology that was feasible for the severely impaired participants to use, large amounts of missing data, and the constantly changing CAPS intervention techniques (19).

\section{Discussion}

Analysis of literature showed an emerging prevalence of behavioural therapies, subsequent to 2000 , that focused on remediating verbal impairment in children diagnosed with autism. After evaluating the papers using the CONSORT style checklist for methodological quality, the collective scores indicate that some existing research exceeds the average of 17 out of 32 . This indicates that some examined studies were comprehensive longitudinal research 
designs that considered design fundamentals, sample size, internal validity, external validity, and generalizability. However, the selected papers failed to provide quantitative justification for much of the validity criteria. Although the majority of the chosen studies scored high on the checklist, limitations and sources of error still exist in current research.

The majority of selected studies utilized a sample size of under twenty participants $(14,15,16,17)$. The sample size limitations resulted in lower statistical power, and thereby less confidence in the study results. Larger sample sizes are more representative of the general target population. This concept has been proven with multiple studies that examine the effect of sample size (20). Using a smaller sample size than the ideal statistical power scenario increases the likelihood of false conclusions. Also, a smaller sample size limits the external validity, and ability to generalize findings for varying levels of autism symptomatology, as variation is not represented among restricted sample sizes (20). A potential solution would be performing meta-analyses to pool the statistical power of various studies examining the same interventions. Having a sample size calculation is important and will assure that the results obtained have the highest statistical power, allowing for generalized conclusions.

Gender bias was commonly observed in many of the studies when choosing a sample population. Although presumably each study included a larger proportion of male participants since autism is generally found to be more prevalent amongst males, there is evidence to suggest that the gender disparity amongst autistic children is contingent on different factors (21). One of these factors is severity of the disorder (21). To combat this limitation, future studies can stratify their participants based on whether they were at a moderate, severe, or profound level of cognitive behavioural abilities, and adjust each group to fit the appropriate sex ratio. Additionally, Wing demonstrates that the sex ratio in childhood autism differs in regards to social, visuo-spatial, and language skills (21). Thus, the determination of the sample's sex ratio in future studies should consider the specific skill set being utilized by the intervention of interest.

Selection bias was introduced by many of the papers studied. As autism is most prevalent in Caucasians, followed by AfricanAmericans, and then by Hispanics, there is evidence to suggest that participants in the studies by Rogers et al., Dethorne et al., and Carter et al., were chosen based off of their ability to fit the racial distribution of autism $(15,16,18,21)$. This indicates that the target populations for these specific studies were not chosen based on prevalence, rather their ability to fit the criteria, hindering the re- sults of the data. Perhaps, moving forward, resources should be allocated to investigating clinical changes in ethnic groups with the highest prevalence rates of autism. Furthermore, the CDC notes that the distribution may not be reflective of the general autistic population, as socioeconomic characteristics can limit patient access to diagnostic centers and treatment measures (22). Due to the necessity of requiring financial support in order to accurately diagnose these populations, many individuals with a low socioeconomic status may have met the criteria for autism, however were unable to participate due to their inability to finance the costs of diagnosis. These studies are limited to populations who can afford the resources available, therefore limiting the research on individuals who fit the criteria, but are financially unable to participate in the study. The lack of a significant study population based on socioeconomic status may alter data conclusions, therefore, it becomes necessary to incorporate these individuals to assure all variables are taken into consideration.

Many of the studies examined lacked a control group, hindering the validity and reliability of the results. For example, the studies conducted by Garcia-Albea et al. and Rogers et al. included cases with no controls $(14,16)$. The absence of baseline data for comparison, particularly when analyzing participant progress, diminished confidence in the results as any changes observed could not be directly attributed to the treatment. Understandably, voluntary participation, in addition to time and fiscal constraints, make implementation of a control group extremely tedious. Studies have noted that therapeutic effects of behavioural therapies are most strongly seen when parallel to randomized control groups (23). A control group allows researchers to analyze whether the treatment in question exceeds the impact of "common factors," or factors apparent in all forms of therapy. Controls would reduce the impact of placebo effects; the natural expectation that any administered therapy should help, compliance issues, and the therapeutic relationship between the psychotherapy provider and receiver. Importantly, analysis must not neglect the natural development of the child, however, it is extremely difficult to differentiate between clinical differences due to natural cognitive development, versus intervention induced changes. Therefore, a control group may also minimize therapist, or environmental effects that alter how the psychotherapy is received, and the outcome it generates $(23,24)$.

Additionally, participants simultaneously underwent external therapies, in addition to the intervention pursued during the study, to address symptoms other than verbal impairments. As these interventions were not standardized, and were potentially unreported, there is no way of examining their influence on the changes 
in symptomatology noticed. More importantly, it is unethical to withhold or standardize treatment provided outside the confines of the experimental protocol, making it difficult to improve the reliability of the results (16). Several of these studies, including Green et al., Casenhiser et al., and Rogers et al., commented that parent-mediated interventions, such as speech therapy techniques employed in the residential setting, were unable to be standardized as the interventions took place in a non-controlled environment $(11,12,16)$. Therefore, therapy sessions may have varied in length and effectiveness, making time reported on parent lead therapy sessions an underestimate. This jeopardized the uniformity of the treatments given and thus, the validity of the results, and is an issue present in all longitudinal data. It may, in fact, be unethical to restrict a participant to only one intervention. To minimize the effect of unmediated treatments interfering with study results, research moving forward should distinguish between an ideal trial and a pragmatic intervention trial designed to inform clinical practice. Studies in the past that have completed an "ideal trial" to determine the efficacy of a therapy should progress their research by conducting pragmatic intervention trials as an added layer of analysis $(24,25)$.

Further research in these five key areas, (sample size, control group, ethnic diversity, external therapies, and parent-mediated interventions,) is imperative to achieving a greater understanding of speech-based behavioural therapies in children with autism. Future studies could reassess the aforementioned interventions using a larger sample size and control groups as a baseline to verify the results. To add merit to the smaller scale studies and their respective therapies, perhaps the reviewed studies could be replicated with these parameters to improve confidence of the results. This may be of greater value rather than conducting several smaller scale studies on interventions that have a high risk of inconclusive and non-generalizable results. Selection bias can be avoided using random participant selection from a wide range of geographic and ethnographic origins. The effects of confounding caused by simultaneous external therapies may be mitigated by keeping extensive records of the therapies participants undergo. This allows for a clearer understanding of the types of confounding effects that may be expected. Parent-mediated interventions may be streamlined by asking parents to maintain detailed records of therapy administration (which can be reviewed by investigators at regular intervals).

As children with autism have a broad range of abilities, future studies should also attempt to group similarly abled children together, as matching methods may strengthen study design. Taking advantage of isolated mathematical or artistic skills and incorpo- rating them into educational tasks for future interventions could increase positive effects on learning since it would increase motivation. This can be seen in the study by Kim et al. which grouped children with similar IQ's, ensuring methods could be tailored to fit the unique needs of differently-abled autistic children (26). Future interventions could be more beneficial if they were personalized to each individual. As seen in the study conducted by GarciaAlbea et al., toys preferred by the participants resulted in increased interactions and better results (14). When a method is formed to suit large groups of children, it doesn't take into account what would be most effective for each individual child, thus decreasing its effectiveness. Therefore, personalization could be a factor in determining the effectiveness of interventions. Understandably, feasibility would be difficult as larger samples would require identification of subgroups with similar profiles.

\section{Conclusion}

Based on the studies examined, study samples in research about behavioural therapies for verbal impairments in autistic children are unrepresentative of the population. They lack control groups, a large enough sample size, and gender, ethnic, and socioeconomic diversity. We also found that papers often neglected to include essential quantitative information that affected their internal and external validity. In order the improve the quality and relevance of research, future studies must include control groups, appropriate sample sizes based on sample size calculations, and samples that are representative of the study population. It is imperative that these steps are considered in the future to validate the results and concretely determine the effects of behavioural therapies.

\section{Acknowledgements}

We would like to acknowledge and thank our initial mentor Jiin Kim, and Dr. Stelios Georgiades and Dr. Steven Taylor for their helpful and thought-provoking feedback. 


\section{References}

1. American Psychiatric Association. (2013). Diagnostic and statistical manual of mental disorders (5th ed.). Washington, DC: Author.

2. What are Autism Spectrum Disorders? [Internet]. Geneva Centre for Autism. Available from: https://www.autism.net/resources/about-autism/40-what-areautism-spectrum-disorders.html [Accessed 15th May 2017].

3. Autism Spectrum Disorder: Causes [Internet]. American Speech-Language-Hearing Association. ASHA. Available from: http://www.asha.org/PRPSpecificTopic .aspx?folderid=8589935303§ion=Causes [Accessed 15th May 2017].

4. Laugeson EA, Park MN. Using a CBT approach to teach social skills to adolescents with autism spectrum disorder and other social challenges: the PEERS ${ }^{\circledR}$ method. Journal of Rational-Emotive \& Cognitive-Behavior Therapy. 2014 Mar 1;32(1):84-97.

5. Tincani M. Moving forward: Positive behavior support and applied behavior analysis. The Behavior Analyst Today. 2007;8(4):492. Available from:doi : http://dx.doi.org/10.1037/h0100635

6. Granpeesheh D, Tarbox J, Dixon DR. Applied behavior analytic interventions for children with autism: a description and review of treatment research. Annals of clinical psychiatry. 2009 Aug 1;21(3):162-73. Available from: https://www.ncbi.nlm.nih.gov/pubmed/19758537 [Accessed 3rd Oct 2017]

7. Beck JS. Cognitive behavior therapy: Basics and beyond. Guilford Press; 2011 Aug 18. Available from: https://books.google.ca/books?hl=en\&lr=\&id=J iAUcHc60cC\&oi=fnd\&pg=PR1\&dq=Beck+JS. + Cognitive+behavior+therapy:+Basics + and + beyond. + Guilford + Press $\% 3 B+2011+$ Aug $+18 . \&$ ots $=0 \mathrm{D}-\mathrm{VQ}$ 9YVCy\&sig=i-_Q3_E-1UqY11IIGtrcLJrzJas\#v=onepage\&q\&f=false [Accessed 3rd October 2017]

8. Wolf MM, Kirigin KA, Fixsen DL, Blase KA, Braukmann CJ. The Teaching-Family Model: A case study in data-based program development and refinement (and dragon wrestling). Journal of Organizational Behavior Management. 1995 Sep 25;15(1-2):11-68. Available from:doi: https://doi.org/10.1300/ J075v15n01_04

9. Tooth L. Quality of Reporting of Observational Longitudinal Research. American Journal of Epidemiology [Internet]. 2005Jan;161(3):280-8. Available from:doi: https://doi.org/10.1093/aje/kwi042

10. National Research Council. Educating children with autism. National Academies Press; 2001 Nov 11.

11. Casenhiser DM, Binns A, Mcgill F, Morderer O, Shanker SG. Measuring and Supporting Language Function for Children with Autism: Evidence from a Randomized Control Trial of a Social-Interaction-Based Therapy. Journal of Autism and Developmental Disorders. 2014. Available from:doi: https://doi. org/10.1007/s10803-014-2242-3

12. Green J, Charman T, McConachie H, Aldred C, Slonims V, Howlin P, Le Couteur A, Leadbitter K, Hudry K, Byford S, Barrett B. Parent-mediated communication-focused treatment in children with autism (PACT): a randomised controlled trial. The Lancet. 2010 Jun 25;375(9732):2152-60. Available from:doi: 10.1016/S0140-6736(10)60587-9.

13. Howlin P, Gordon RK, Pasco G, Wade A, Charman T. The effectiveness of Picture Exchange Communication System (PECS) training for teachers of children with autism: a pragmatic, group randomised controlled trial. Journal of Child Psychology and Psychiatry [Internet]. 2007 [cited 2017Oct4];48(5):47381. Available from:doi: 10.1111/j.1469-7610.2006.01707.x

14. Garcia $\square$ Albea E, Reeve SA, Brothers KJ, Reeve KF. Using audio script fading and multiple $\square$ exemplar training to increase vocal interactions in children with autism. Journal of Applied Behavior Analysis. 2014. Available from:doi: 10.1002/jaba.125.

15. DeThorne L, Betancourt MA, Karahalios K, Halle J, Bogue E. Visualizing Syllables: Real-Time Computerized Feedback Within a Speech-Language Intervention. Journal of Autism and Developmental Disorders. 2015. Available from:doi: https://doi.org/10.1007/s10803-014-2274-8

16. Rogers SJ, Hayden D, Hepburn S, Charlifue-Smith R, Hall T, Hayes A. Teaching young nonverbal children with autism useful speech: A pilot study of the Denver model and PROMPT interventions. Journal of Autism and Developmental Disorders. 2006 Nov 1;36(8):1007-24 Available from:doi: 10.1007/ s10803-006-0142-x

17. Charlop-Christy MH, Kelso SE. Teaching children with autism conversational speech using a cue card/written script program. Education and Treatment of Children. 2003 May 1:108-27. Available from:http://www.jstor.org/stable/42899741[Accessed 3rd Oct 2017].

18. Carter AS, Messinger DS, Stone WL, Celimli S, Nahmias AS, Yoder P. A randomized controlled trial of Hanen's 'More Than Words' in toddlers with early autism symptoms. Journal of Child Psychology and Psychiatry and Allied Disciplines. 2011 Jul;52(7):741-752. Available from:doi: 10.1111/j.14697610.2011.02395.x

19. Studer, N., Gundelfinger, R., Schenker, T., \& Steinhausen, H. (2017). Implementation of early intensive behavioural intervention for children with autism in Switzerland. BMC Psychiatry, 17(1). Available from:doi: https://doi.org/10.1186/s12888-017-1195-4

20. Faber J, Fonseca LM. How sample size influences research outcomes. Dental press journal of orthodontics. 2014 Aug;19(4):27-9.

21. Wing L. Sex ratios in early childhood autism and related conditions. Psychiatry research. 1981 Oct 1;5(2):129-37.

22. CDC. Autism Spectrum Disorder (ASD) [Internet]. Autism and Developmental Disabilities Monitoring (ADDM) Network. Centers for Disease Control and Prevention; 2016. Available from: https://www.cdc.gov/ncbddd/autism/addm.html [Accesseed 5 October 2017]

23. Safer DL, Hugo EM. Designing a control for a behavioral group therapy. Behavior therapy. 2006 Jun 1;37(2):120-30.

24. Wampold BE. How important are the common factors in psychotherapy? An update. World Psychiatry. 2015 Oct 1;14(3):270-7.

25. Ford I, Norrie J. Pragmatic trials. New England Journal of Medicine. 2016 Aug 4;375(5):454-63. Available from: 10.1056/NEJMra1510059

26. Kim, E., Kyeong, S., Cheon, K., Park, B., Oh, M., \& Chun, J. et al. (2016). Neural responses to affective and cognitive theory of mind in children and adolescents with autism spectrum disorder. Neuroscience Letters, 621, 117-125. Available from:doi: 10.1016/j.neulet.2016.04.026 